TITLE:

\title{
Metal nanoparticle precipitation in periodic arrays in Au2O-doped glass by two interfered femtosecond laser pulses
}

\section{$\operatorname{AUTHOR}(\mathrm{S}):$}

Qu, SL; Qiu, JR; Zhao, CJ; Jiang, XW; Zeng, HD; Zhu, CS; Hirao, K

\section{CITATION:}

Qu, SL ... [et al]. Metal nanoparticle precipitation in periodic arrays in Au2O-doped glass by two interfered femtosecond laser pulses. APPLIED PHYSICS LETTERS 2004, 84(12): 20462048

\section{ISSUE DATE:}

2004-03-22

URL:

http://hdl.handle.net/2433/39664

\section{RIGHT:}

Copyright 2004 American Institute of Physics. This article may be downloaded for personal use only. Any other use requires prior permission of the author and the American Institute of Physics. 


\title{
Metal nanoparticle precipitation in periodic arrays in $\mathrm{Au}_{2} \mathrm{O}$-doped glass by two interfered femtosecond laser pulses
}

\author{
Shiliang $\mathrm{Qu}^{\mathrm{a})}$ \\ Photon Craft Project, Shanghai Institute of Optics and Fine Mechanics, Chinese Academy of Sciences, \\ and Japan Science and Technology Agency, Shanghai 201800, China and Department of Applied Physics, \\ Harbin Institute of Technology at Weihai, Weihai 264209, China \\ Jianrong Qiu, Chongjun Zhao, Xiongwei Jiang, Huidan Zeng, and Congshan Zhu \\ Photon Craft Project, Shanghai Institute of Optics and Fine Mechanics, Chinese Academy of Sciences, \\ and Japan Science and Technology Agency, Shanghai 201800, China \\ K. Hirao \\ Department of Material Chemistry, Graduate School of Engineering, Kyoto University, Sakyo-ku, \\ Kyoto 606-8501, Japan
}

(Received 17 September 2003; accepted 30 January 2004)

\begin{abstract}
We report on the precipitation control of $\mathrm{Au}$ nanoparticles in periodic arrays in silicate glass. $\mathrm{Au}_{2} \mathrm{O}$-doped glass samples were first irradiated by two $800 \mathrm{~nm}$ interfered femtosecond laser pulses at room temperature and then heat treated at $550{ }^{\circ} \mathrm{C}$ for the $\mathrm{Au}$ nanoparticle precipitation in the laser irradiation areas. One-dimensional periodic arrays of the Au nanoparticles were controlled by changing the pulse energy and the incident angle between the interfered laser pulses. The smallest dimension in the obtained arrays was a width of $300 \mathrm{~nm}$. The mechanism of the metal nanoparticle precipitation by this technique was discussed. Only two pulses are required to encode these periodic microstructures, which are applicable to emerging nanostructure devices such as optical memory with ultrahigh storage density, micrograting with high diffractive efficiency and integrative micro-optical switches. (C) 2004 American Institute of Physics. [DOI: 10.1063/1.1688004]
\end{abstract}

Noble metal nanoparticle-contained glasses exhibit large third-order nonlinear susceptibility and ultrafast nonlinear response due to the local field effect near surface plasmon resonance and quantum size effect. ${ }^{1-3}$ They are expected to be promising materials for an ultrafast optical limiter and all-optical switches. ${ }^{3,4}$ But then, nanoparticles must be arranged into well-defined and space-selective microstructures in the glasses in order to build integrated systems. In recent years, many studies have been carried out on the fabrication of nanoparticle-doped glasses. ${ }^{5-9}$ However, they are not effective preparation methods from which nanoparticles constituted periodic microstructures can be space-selectively made and controlled with holographic techniques.

In this letter we demonstrate the formation of onedimensional microarrays constituted with $\mathrm{Au}$ nanoparticles in $\mathrm{Au}_{2} \mathrm{O}$-doped glasses induced by two interfered femtosecond laser pulses followed by successive heat treatment. The spacing and width of the microarrays can be controlled by changing the incident angle between the two interfered pulses and the laser pulse energy. This method should be useful not only for practical applications in the fabrication of integrative all-optical devices, but also for controlling nucleation process.

We selected a typical silicate glass composed of $70 \mathrm{SiO}_{2} \cdot 20 \mathrm{Na}_{2} \mathrm{O} \cdot 10 \mathrm{CaO}$ doped with $0.1 \mathrm{Au}_{2} \mathrm{O}(\mathrm{mol} \%)$. Reagent grade $\mathrm{SiO}_{2}, \mathrm{CaCO}_{3}, \mathrm{Na}_{2} \mathrm{CO}_{3}$, and $\mathrm{AuCl}_{3} \cdot \mathrm{HCl}$ - $4 \mathrm{H}_{2} \mathrm{O}$ were used as starting materials. An approximately 40 $\mathrm{g}$ batch was mixed and placed into a platinum crucible. Melt-

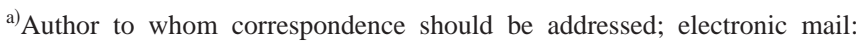
slqu1@yahoo.com.cn
}

ing was carried out in an electric furnace at $1550{ }^{\circ} \mathrm{C}$ for $1 \mathrm{~h}$. The glass sample was obtained by quenching the melt to room temperature. The sample thus obtained was transparent and colorless. The annealed sample was cut and polished, and then subjected to experiments.

Figure 1 illustrates the geometry used for the microstructure fabrication in the glass samples. A regeneratively amplified femtosecond pulse from a Ti:sapphire laser (Spectraphysics), with $800 \mathrm{~nm}, 120 \mathrm{fs}$, and a repetition rate of $1-1000 \mathrm{~Hz}$, was split into two equal energy beams that were redirected at approximately equal angles on the surface of the sample. The two beams, with diameters of $\sim 5 \mathrm{~mm}$, were focused by lenses with focal length of $10 \mathrm{~cm}$ to give a spot

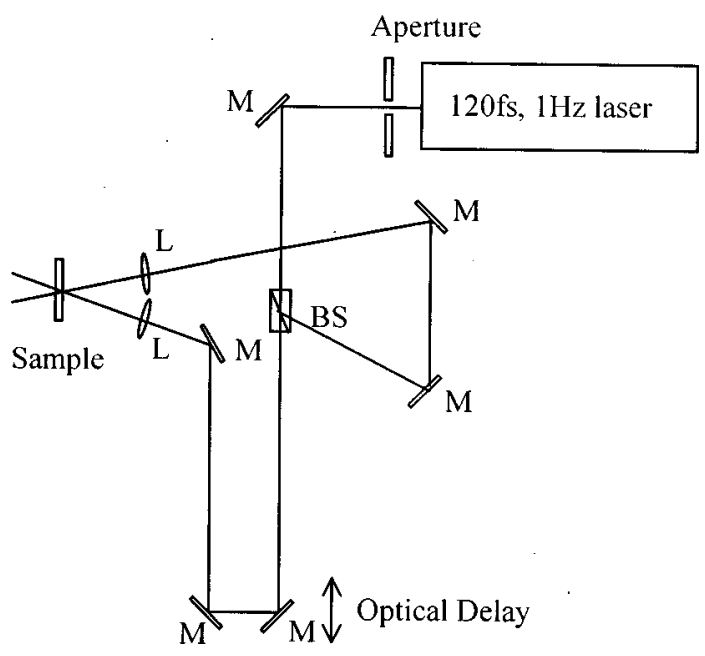

FIG. 1. Setup for holographic encoding by femtosecond laser pulses. 


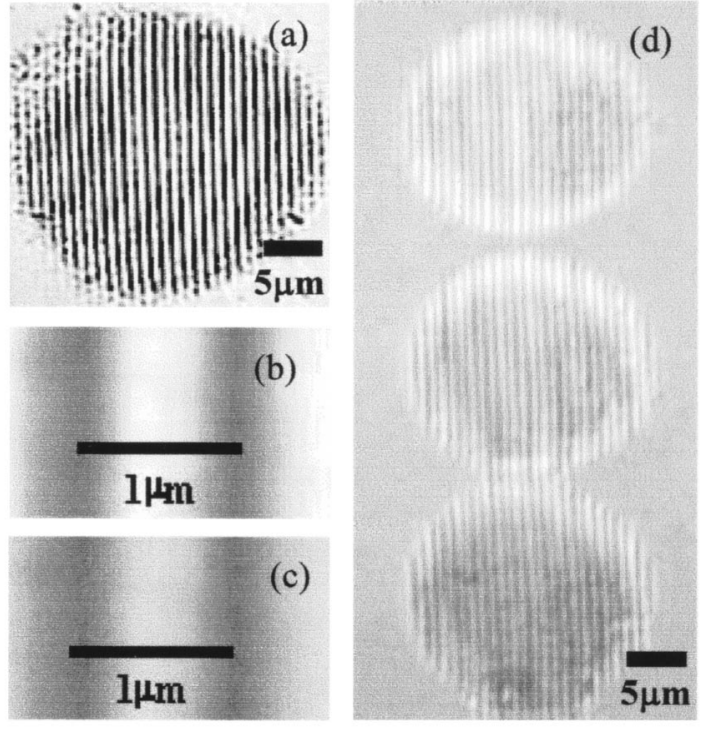

FIG. 2. Optical microscopic photos of Au nanoparticles precipitaion in periodic arrays in silicate glass (i.e., microgratings), taken by a $100 \times$ transilluminated optical microscope. (a) Energy is $30 \mu \mathrm{J}$ per pulse. (b) Magnified view of (a). (c) Energy is $38 \mu \mathrm{J}$ per pulse. (d) Part of a group of microgratings.

size of $\sim 35 \mu \mathrm{m}$ at the focal plane. A time-delay device was used to adjust the timing of pulse collisions. We achieved a time superposition of pulses between the two incident beams by adjusting the time-delay device and observing their second harmonic generated from a BBO crystal. After the optical paths were adjusted to give perfect overlap of the two beams both spatially and temporarily, we regulated the sample to ensure the surface normal to the perpendicular bisector of the two incident beams. The surface will be referred to as the laser interfering plane in the following.

The two incident beams were first focused onto the front surface of $\mathrm{Au}_{2} \mathrm{O}$-doped glass to optimize the incident pulse energy. In the case of sufficiently high energy, the two coherent beams can induce periodic ablation, forming a grating in the glass. Herein, we reduced the energy to a certain lower level at which the two coherent beams cannot directly induce periodic ablation, and the grating can only be formed after heat treating the sample at $550{ }^{\circ} \mathrm{C}$ for $1 \mathrm{~h}$. Such grating was constituted by the laser-heating induced Au nanoparticle precipitation in the $\mathrm{Au}_{2} \mathrm{O}$-doped glass. In our experiments, this lower pulse energy was selected to be $\sim 30$ and $38 \mu \mathrm{J}$ for comparison, and the colliding angle $\theta$ between the two incident beams was fixed at $\sim 45^{\circ}$.

With a halogen lamp across the laser irradiated and heat treated sample, the micrograting with a periodic and violetcolored array can be clearly observed with the aid of a $100 \times$ transilluminated optical microscope whose objective was focused onto the surface of the sample, as shown in Fig. 2(a). The period $d$ of the micrograting was $\sim 1 \mu \mathrm{m}$, agreeing with the value calculated from the colliding angle $\theta$ and the laser wavelength $\lambda$ according to the formula $d$ $=\lambda /[2 \sin (\theta / 2)]$. The widths of violet-colored lines for pulse energies of 30 and $38 \mu \mathrm{J}$ were estimated to be $\sim 300$ and 500 $\mathrm{nm}$, respectively, through a magnified view of Fig. 2(a), as shown in Figs. 2(b) and 2(c).

To confirm the $\mathrm{Au}$ nanoparticle precipitation in the Downloaded 30 May 2007 to 130.54.110.22. Redistribution subject

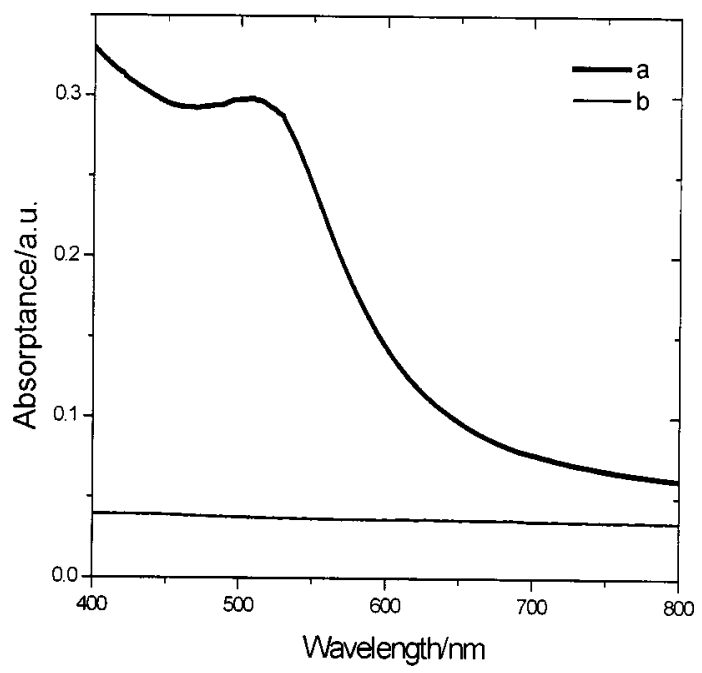

FIG. 3. Absorption spectra of the glass samples after holographic irradiation by femtosecond laser pulses with (a) and without (b) heat treatment.

violet-colored lines, we moved the sample at a constant speed, which was synchronized with the laser pulse repetition rate $(1 \mathrm{~Hz})$. Each coherent spot of $\sim 35 \mu \mathrm{m}$ in diameter was encoded by a single shot of $38 \mu \mathrm{J}$. The sample was then heat treated for $1 / 2 \mathrm{~h}$ at $550{ }^{\circ} \mathrm{C}$. Thus a new grating (1 $\times 1 \mathrm{~mm}^{2}$ ) in the glass sample was fabricated. Figure 2(d) shows a part of the new grating. The absorption spectrum of the grating was measured by a spectrophotometer (JASCO V-570) as shown in Fig. 3. Apparently, a weak peak occurs around $508 \mathrm{~nm}$, which is induced by the surface plasmon resonance of An nanoparticles in the glass. The Au nanoparticles with $\sim 3 \mathrm{~nm}$ average size in the glass were observed from a transmitted electronic microscopy image as shown in Fig. 4. However, we observed neither absorption peak in the range of 500-600 nm as seen in Fig. 4, nor Au nanoparticles constituted micrograting in the glass sample irradiated only by the interfered pulses but not heat treated. This indicates that the Au nanoparticles can be precipitated in the periodic one-dimensional arrays in the glass through the irradiation of two coherent beams with the aid of heat treatment.

In the light of the nanoparticles size and the absorbance of the micrograting, the volume density of Au nanoparticles in the micrograting was estimated to be $\sim 10^{-5}$. In addition,

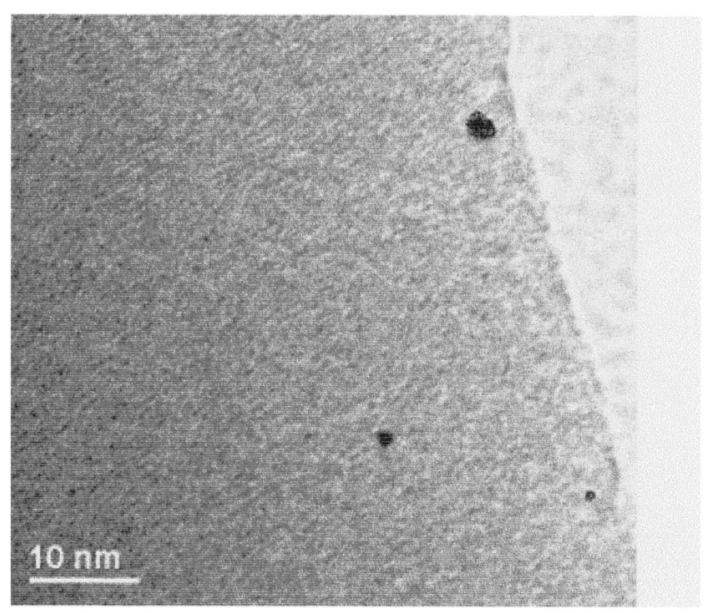

FIG. 4. TEM image of the glass sample after holographic irradiation by femtosecond laser pulses and heat treatment.

o AIP license or copyright, see http://apl.aip.org/apl/copyright.jsp 
the interfered optical electric field is sinusoidal. Therefore, the distribution of Au nanoparticles in depth is quasisinusoidal, which should be similar with the structure of surface relief grating. ${ }^{10}$ The density and depth distribution of $\mathrm{Au}$ nanoparticles can not affect the size of the micrograting, but the optical intensity of the micrograting improves as the density and depth increase. When we used a low intensity $\left(0.6 \mathrm{~mW} / \mathrm{cm}^{2}\right)$ beam of $632.8 \mathrm{~nm}$ from a He-Ne laser as a probe, the first order diffraction efficiency of the micrograting reaches up to $48 \%$. Otherwise, it is $23 \%$ for the grating obtained on the glass from ablation by the interfered laser irradiation with a pulse energy of $80 \mu \mathrm{J}$.

We also carried out experiments to clarify the mechanism of the formation of the micrograting. As the femtosecond pulses were focused to the glass, supercontinuum white light was observed, which arises from self-phase modulation of the laser beam. The intensity for the fundamental wave and supercontinuum white light is high enough for multiphoton ionization of glass matrix. ${ }^{11}$ Therefore, the active electrons and holes can be created in the glass by laser irradiation through the multiphoton ionization. In this case, part of the electrons are driven out of the $2 p$ orbital of the nonbridging oxygen in the $\left[\mathrm{SiO}_{4}\right]$ polyhedron via the multiphoton absorption of the incident photon. $\mathrm{Au}^{+}$captures the electron to form an $\mathrm{Au}$ atom. Au atoms aggregated to form nanoparticles after the heat treatment. ${ }^{5}$ In addition, in the unirradiated $\mathrm{Au}_{2} \mathrm{O}$-doped glass sample, $\mathrm{Au}$ nanoparticle precipitation was not found even heating the sample at $580^{\circ} \mathrm{C}$ for $1 \mathrm{~h}$. Therefore, the reduction of $\mathrm{Au}^{+}$to atom by the interfered irradiation is essential in forming $\mathrm{Au}$ nanoparticles, and the atom acts as a crystal nucleus in the crystal growth progress during the heat treatment. Also, no change was observed in the absorption spectrum of the Au nanoparticles precipitated glass sample at room temperature, even for 5 months. This indicates that the precipitated nanoparticles are very stable at room temperature.

Similarly, we carried out $\mathrm{Ag}, \mathrm{Cu}$, and $\mathrm{Pd}$ nanoparticle precipitation in periodic arrays in glasses when the colliding angle $\theta$ between the two incident beams was fixed at $\sim 90^{\circ}$. Three kinds of lines of yellow, dark red, and dark gray colors in the microgratings can be seen by a $100 \times$ transilluminated optical microscope. The period $d$ of the resulting gratings was $\sim 0.6 \mu \mathrm{m}$. Thus, we can adjust the period of the microgratings of noble metal nanoparticles through changing the colliding angle $\theta$, and adjust the width of the colored line by changing the laser pulse energy.

In conclusion, we have observed noble metal nanoparticles precipitated in periodic one-dimensional arrays (i.e., microgratings) in silicate glasses by two interfered femtosecond laser pulses with the aid of heat treatment. Encoding the micrograting of metal nanoparticles by the present holographic technique has not been reported to date. This technique has potential for the fabrication of micrograting with high diffractive efficiency as diffractive elements. Moreover, if one recording beam is modulated as a signal beam, optical information can be stored in transparent materials by this holographic method. A study on metal nanoparticle precipitation in glasses by interfered multibeams irradiation is in progress.

The authors gratefully acknowledge the financial support for this work by the National Natural Science Foundation of China (No. 50125258), the Shanghai Committee of Science and Technology of China (0259nm055), and the Foundation of HIT at Weihai of China [HIT(WH).2002.28].

${ }^{1}$ L. Yang, D. H. Osborne, R. F. Haglund, Jr., R. H. Magruder, C. W. White, R. A. Zuhr, and H. Hosono, Appl. Phys. A 62, 403 (1996).

${ }^{2}$ H. B. Liao, R. F. Xiao, J. S. Fu, H. Wang, K. S. Wong, and G. K. L. Wong, Opt. Lett. 23, 388 (1998)

${ }^{3}$ S. Qu, C. Zhao, X. Jiang, G. Fang, Y. Gao, H. Zeng, Y. Song, J. Qiu, C. Zhu, and K. Hirao, Chem. Phys. Lett. 368, 352 (2003).

${ }^{4}$ R. Philip and G. R. Kumar, Phys. Rev. B 62, 13160 (2000).

${ }^{5}$ J. Qiu, M. Shiral, T. Nakaya, J. Si, X. Jiang, C. Zhu, and K. Hirao, Appl. Phys. Lett. 81, 3040 (2002).

${ }^{6}$ J. Sasai and K. Hirao, J. Appl. Phys. 89, 4548 (2001).

${ }^{7}$ E. Valentin, H. Bernas, C. Ricolleau, and F. Creuzet, Phys. Rev. Lett. 86, 99 (2001).

${ }^{8}$ H. Homfmeister, S. Thiel, M. Dubiel, and E. Schurig, Appl. Phys. Lett. 70, 1694 (1997)

${ }^{9}$ S. Chen, T. Akai, K. Kadono, and T. Yazawa, Appl. Phys. Lett. 79, 3687 (2001).

${ }^{10}$ K. Kawamuara, N. Sarukura, M. Hirano, N. Ito, and H. Hosono, Appl. Phys. Lett. 79, 1228 (2001).

${ }^{11}$ B. C. Stuart, M. D. Feit, A. M. Rubenchik, B. Shore, and M. D. Perry, Phys. Rev. Lett. 74, 224 (1995). 